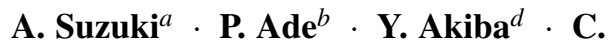

$\operatorname{Aleman}^{l}$. K. Arnold ${ }^{l}$. M. Atlas ${ }^{l}$. D.

Barron $^{l}$ · J. Borrill ${ }^{g}$ - S. Chapman ${ }^{c}$. Y.

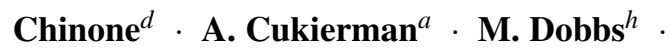

T. Elleflot ${ }^{l}$ - J. Errard $^{g}$ - G. Fabbian ${ }^{f}$. G.

Feng $^{l} \cdot$ A. Gilbert ${ }^{h} \cdot$ W. Grainger ${ }^{b} \cdot \mathbf{N}$.

Halverson $^{m} \cdot$ M. Hasegawa ${ }^{d} \cdot$ K. Hattori ${ }^{d}$.

M. Hazumi ${ }^{d}$ - W. Holzapfel ${ }^{a}$. Y. Hori ${ }^{d} \cdot$ Y.

Inoue $^{d}$ - G. Jaehnig ${ }^{m}$ - N. Katayama ${ }^{e}$ - B.

Keating ${ }^{l} \cdot$ Z. Kermish ${ }^{k} \cdot$ R. Keskitalo ${ }^{g}$.

T. Kisner ${ }^{g}$ - A. Lee ${ }^{a}$ - F. Matsuda ${ }^{l}$. T.

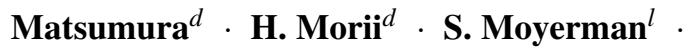

M. Myers $^{a}$ - M. Navaroli ${ }^{l}$ - H. Nishino ${ }^{e}$ - T.

Okamura $^{d}$. C. Reichart ${ }^{a}$. P. Richards ${ }^{a}$.

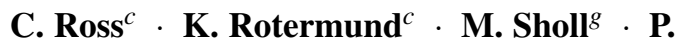

Siritanasak $^{l}$. G. Smecher ${ }^{h}$. N. Stebor ${ }^{l}$.

R. Stompor ${ }^{l} \cdot$ J. Suzuki ${ }^{d} \cdot$ S. Takada $^{i} \cdot$ S.

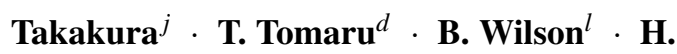

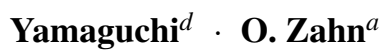

\title{
The POLARBEAR-2 Experiment
}

07.15.2013

\footnotetext{
${ }^{a}$ Department of Physics, University of California, Berkeley

E-mail: asuzuki@berkeley.edu

${ }^{b}$ School of Physics and Astronomy, University of Cardiff

${ }^{c}$ Department of Physics and Atmospheric Science, Dalhousie University

${ }^{d}$ High Energy Accelerator Research Organization

${ }^{e}$ Kavli Institute of Physics and Mathematics of the Universe

${ }^{f}$ Laboratoire Astroparticule and Cosmologie
} 


\begin{abstract}
We present an overview of the design and development of the POLARBEAR2 experiment. The POLARBEAR-2 experiment is a Cosmic Microwave Background polarimetry experiment, which aims to characterize the small angular scale B-mode signal due to gravitational lensing and search for the large angular scale B-mode signal from inflationary gravitational waves. The experiment will have a $365 \mathrm{~mm}$ diameter multi-chroic focal plane filled with 7,588 polarization sensitive antenna-coupled Transition Edge Sensor bolometers and will observe at $95 \mathrm{GHz}$ and $150 \mathrm{GHz}$. The focal plane is cooled to 250 milli-Kelvin. The bolometers will be read-out by SQUIDs with $32 x$ frequency domain multiplexing. The experiment will utilize high purity alumina lenses and thermal filters to achieve the required high optical throughput. A continuously rotating, cooled half-wave plate will be used to give stringent control over systematic errors. The experiment is designed to achieve a noise equivalent temperature of $5.7 \mu \mathrm{K} \sqrt{s}$, and this allows us to constrain the signal from the inflationary primordial gravitational corresponding to a tensor-to-scalar ratio of $r=0.01(2 \sigma)$. POLARBEAR-2 will also be able to put a constraint on the sum of neutrino masses to $90 \mathrm{meV}(1 \sigma)$ with POLARBEAR-2 data alone and $65 \mathrm{meV}(1 \sigma)$ when combined with the Planck satellite. We plan to start observations in 2014 in the Atacama Desert in Chile.
\end{abstract}

Keywords Cosmic Microwave Background, Inflation, Lensing, Polarization, B-mode

\title{
1 Introduction
}

Precise measurements of the Cosmic Microwave Background (CMB) temperature anisotropy put tight constraints on cosmological parameters. In the past decade, CMB experiments have shown that a small fraction of the CMB's intensity is

${ }^{g}$ Lawrence Berkeley National Laboratory

${ }^{h}$ McGill University

${ }^{i}$ National Institute for Fusion Science

${ }^{j}$ Department of Physics, Osaka University

${ }^{k}$ Department of Physics, Princeton University

${ }^{l}$ Department of Physics, University of California, San Diego

${ }^{m}$ Department of Physics, University of Colorado, Boulder 
polarized ${ }^{8}$. Measurement of the even-parity polarization pattern (E-mode polarization) agrees with the expectation from the temperature anisotropy measurements ${ }^{\text {? }}$. We also expect an even smaller fraction of the CMB to have an oddparity polarization pattern (B-mode polarization). The B-mode polarization has two sources. Weak gravitational lensing from large scale structure mixes the $\mathrm{E}-$ mode pattern, converting some of it to B-mode polarization pattern ${ }^{5}$. The B-mode signal from weak gravitational lensing is expected to peak around ten-arcmin angular scales. B-modes are also sourced by primordial gravitational waves. ${ }^{13}$. Many inflationary models predict the existence of a B-mode signal at approximately two-degree angular scales. Detection or improved upper limits on the primordial B-modes will put a tight constraint on inflationary models and on the energy scale of the inflaton field. The intensity of the inflationary B-mode signal is expected to be at least four orders of magnitude lower than the temperature anisotropy. Polarized foregrounds, synchrotron radiation and dust emission, from our galaxy can confuse the measurement ${ }^{2}$. Thus, we need to design a highly sensitive experiment that has tight control over systematic errors including broad frequency coverage for foreground mitigation. In this proceeding, we describe how the design of the POLARBEAR-2 experiment achieves those goals.

\section{Project Overview}

The POLARBEAR-2 experiment will observe from the James Ax observatory at an altitude of 5,200 meters on Cerro Toco in the Atacama Desert. This site has a median precipitable water vapor of $1.5 \mathrm{~mm}$. The POLARBEAR-2 receiver will be mounted on a telescope with the same design as the Huan Tran Telescope (HTT) that is currently observing with the POLARBEAR-1 receiver ${ }^{7,1}$. The HTT features an offset Gregorian design meeting the Mizuguchi-Dragone condition, and co-moving baffles minimize instrumental polarization and sidelobes. A 3.5 meter primary mirror produces a 3.5 -arcmin $(5.2$-arcmin) FWHM beam at $150 \mathrm{GHz}$ (95 GHz). We plan to cover $20 \%$ of the sky for three years with an instantaneous array sensitivity of $5.7 \mu \mathrm{K} \sqrt{s}$. Assuming $18 \%$ observation efficiency, we will achieve $10 \mu \mathrm{K}$-arcmin sensitivity. As shown in Figure 1, POLARBEAR-2 will be able to constrain on a signal from the inflationary primordial gravitational 


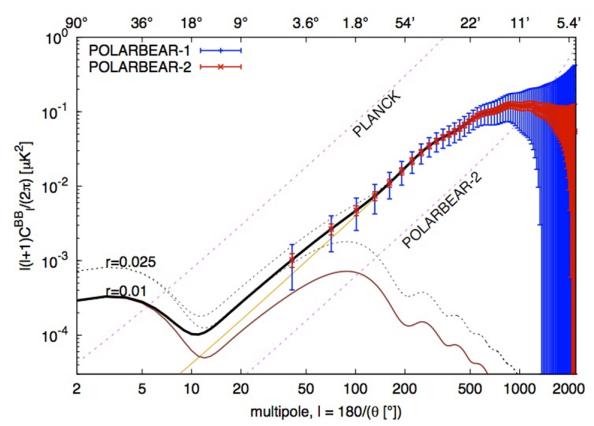

Fig. 1 (Color online) Projected sensitivity of POLARBEAR-2 and Planck. Black solid (blue dot) line is the expected B-mode signal corresponding to $r=0.01(0.025)$. The orange line is the B-mode signal from weak gravitational lensing. The purple solid line (black dot line) is the B-mode signal from the primordial gravitational wave with $r=0.01(0.025)$.

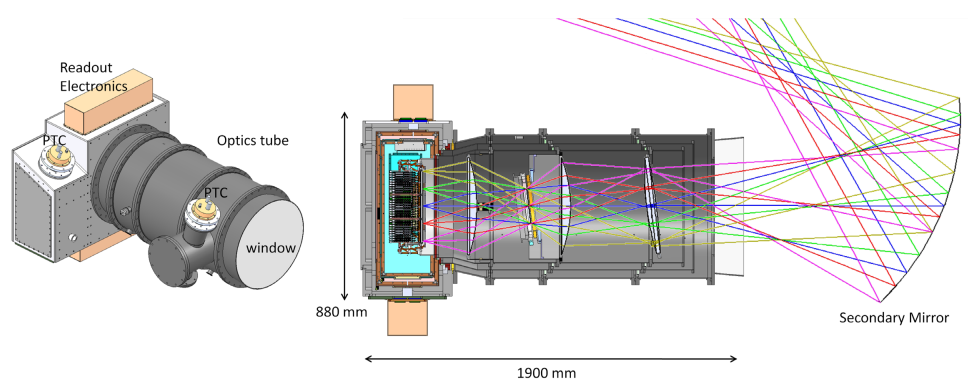

Fig. 2 (Color online) CAD drawing of the POLARBEAR-2 receiver (left), and cross sectional view of the POLARBEAR-2 receiver with overlaid ray traces (right)

waves corresponding to a tensor-to-scalar ratio of $r=0.01$ ( $2 \sigma$ C.L.). Using the weak gravitational lensing signal, the experiment will be able to put a constraint on the sum of neutrino masses to $90 \mathrm{meV}(1 \sigma$ C.L.) and $65 \mathrm{meV}(1 \sigma$ C.L.) when data is combined with Planck data ${ }^{16}$. In simulations, we find that we can subtract the predicted dust emission on our patches (assuming a conservative 10\% dust polarization) with only an approximately $10 \%$ impact on the accuracy for measuring $r$. 


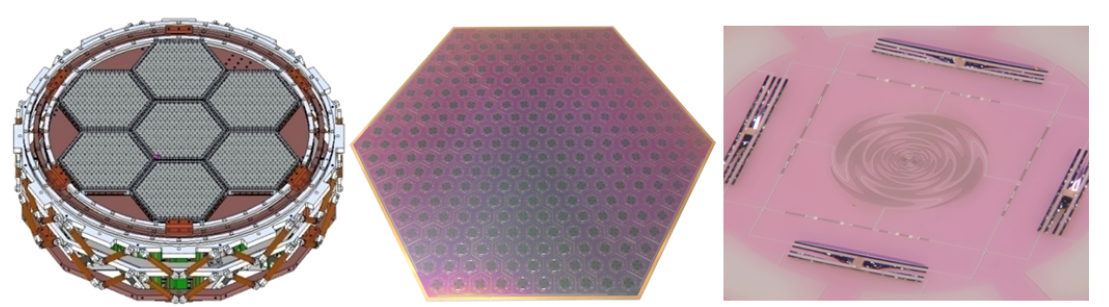

Fig. 3 (Color online) CAD drawing of the $365 \mathrm{~mm}$ focal plane tower (left). Three temperature stages (250 milli-Kelvin, 350 milli-Kelvin, 2 Kelvin) are separated by vespel support structures. Photograph of a single detector array wafer (center). Each wafer is $130 \mathrm{~mm}$ across with 271 pixels. Photograph of a single detector (right) showing the sinuous antenna, a broadband antenna. Diplexer filters surround the antenna. Four dark rectangles are TES bolometers.

\section{Instrument}

A cross-sectional view of the POLARBEAR-2 receiver is shown in Figure 2. The receiver is 1.9 meters long, 1.2 meters wide and 0.88 meters tall. Two Cryomech PT415 pulse-tube coolers cool the receiver. Each cooler provides 50 Kelvin and 4 Kelvin stages. Both coolers are tilted by 21 degrees with respect to the optics tube to have optimal performance when the telescope is scanning at 45 degrees elevation. One pulse-tube cooler is placed near the window of the optics tube to efficiently reduce thermal emission. Another pulse-tube cooler is placed near the focal plane to cool the focal plane and the readout electronics. Annealed 6-N aluminum strips were epoxied to thr receiver shells to increase the thermal conductivity of the receiver. A three-stage helium sorption refrigerator cools the focal plane tower with 2 Kelvin, 350 milli-Kelvin and 250 milli-Kelvin stages.

The optics has a field of view of $4.8^{\circ 11}$. To reduce the thermal loading from the $50 \mathrm{~cm}$ diameter window in the optics tube, we use high purity (99.9\%) alumina as an infra-red filter. Alumina absorbs infrared photons effectively, yet it is transparent at milli-meter waves. Alumina also has three orders of magnitude better thermal conductivity at 100 Kelvin compared to plastic materials that were commonly used as dielectric filters ${ }^{6}$.

Three lenses are fabricated from high purity alumina. The high dielectric constant of alumina $\left(\varepsilon_{r}=9.9\right)$ allows an optics design with a large field of view. High purity alumina also has low loss $(\tan \delta \approx 0.0001)$, and its high thermal conductiv- 
ity helps the overall cryogenic performance. However, the high dielectric constant of alumina requires anti-reflection coating to minimize reflection at the dielectric boundary. Since POLARBEAR-2 observes at $95 \mathrm{GHz}$ and $150 \mathrm{GHz}$ simultaneously, the anti-reflection coating on the lens must cover a wide frequency range. We developed a two-layer epoxy-based antireflection coating ${ }^{15,14}$.

We place a 4 Kelvin cold stop and an achromatic half-wave plate at the aperture. The cold stop is designed for $\mathrm{F} / \#=1.9$ optics. The achromatic half-wave plate is made from stacks of sapphire crystals. The half-wave plate rotates continuously on a superconducting bearing to modulate the polarized signal in order to reduce systematic error from optics ${ }^{9,10}$.

The focal plane is shown in Figure 3. A $365 \mathrm{~mm}$ diameter focal plane tower houses seven detector array modules. Each module has a hexagonal detector array wafer and readout electronics. Readout electronics sits behind the detector array in order to use the focal plane area efficiently. The detector array was fabricated on a $150 \mathrm{~mm}$ wafer at the Berkeley nanofabrication laboratory. Each wafer has 271 dual linear polarized pixels that simultaneously detect $95 \mathrm{GHz}$ and $150 \mathrm{GHz}$ band. Each pixel has a lens coupled broadband antenna that couples the optical signal to a RF circuit on the wafer. Bandpass filters on the wafer split the signal into two separate bands, and then transition edge sensor (TES) bolometers detects the signal ${ }^{12,15,14}$. The focal plane's 7,588 bolometers provide high sensitivity of $5.7 \mu \mathrm{K} \sqrt{s}$.

We use frequency multiplexed Superconducting Quantum Interference Device (SQUID) amplifiers to read-out TES bolometers. A schematic drawing of a readout chain is shown in Figure 4. A high multiplexing factor allows us to read-out of many detectors without thermally loading the focal plane. Each SQUID uses approximately a $\mathrm{MHz}$ of bandwidth to read-out the 32 TES bolometers. High frequency read-out can increase phase delay in the feedback loop and parasitic impedance of the read-out circuit. We use a digital active nulling technology that actively corrects for the phase delay and reduces parasitic inductance from circuit elements between the bias resistor and the SQUID ${ }^{3,4}$. We fabricated interdigitated capacitors with niobium traces on a high resistivity $(>10 \mathrm{~K} \Omega / \mathrm{cm})$ silicon wafer to reduce parasitic resistance from capacitors. The interdigitated capacitors 


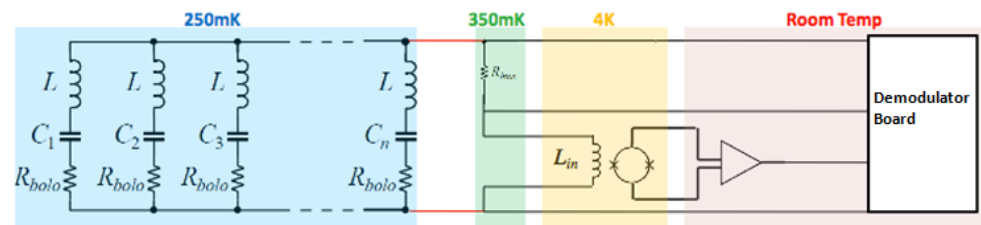

Fig. 4 (Color online) Schematic drawing of the read-out chain. Lithographed inductors and capacitors are in series with bolometers to select frequency channels. Niobium-titanium transmission line thermally isolates 250 milli-Kelvin stage (red line). Bias resistor is placed at 350 milli-Kelvin to minimize physical distance between bias resistor and the focal plane.

have less than $100 \mathrm{~m} \Omega$ parasitic resistance at $3 \mathrm{MHz}$. The capacitors achieve subpercent capacitance accuracy that allows consistent frequency spacing. We also fabricated a niobium-titanium parallel plate transmission line for the 250 milliKelvin to 350 milli-Kelvin connection. Niobium-titanium provides thermal isolation, while the high width-to-height ratio of the parallel plate transmission line provides low inductance per length $(\approx 1 \mathrm{nH} / \mathrm{cm})$.

\section{Conclusions}

The POLARBEAR-2 experiment is designed to measure the CMB's B-mode polarization with high sensitivity. The stringent control of systematic errors, large optical throughput, and high detector count bring new challenges to the experiment. We have addressed these challenges with the unique use of materials, a multichroic detector design, and a new digital electronics design. Currently we are testing many of the components described here. POLARBEAR- 2 is scheduled to deploy in 2014 to Atacama, Chile for 3 years of observations.

Acknowledgements We acknowledge support from the MEXT Kahenhi grant 21111002, NSF grant AST-0618398, NASA grant NNG06GJ08G, The Simons Foundation, Natural Sciences and Engineering Research Council, Canadian Institute for Advanced Research and the CONICYT. Detectors were fabricated at Berkeley nanofabrication laboratory.

\section{References}

1. The POLARBEAR Cosmic Microwave Background Polarization Experi- 
ment,. Journal of Low Temperature Physics, 2013 Submitted.

2. James Bock et al. Task force on cosmic microwave background research. 2006.

3. Tijmen de Haan et al. Improved performance of tes bolometers using digital feedback. pages 84520E-84520E-10, 2012.

4. K. Hattori et al. Adaption of frequency-domain readout for transition edge sensor bolometers for the polarbear- 2 cosmic microwave background experiment. Proceedings for VCI2013, 2013.

5. Wayne $\mathrm{Hu}$ et al. Mass reconstruction with $\mathrm{cmb}$ polarization. Astrophys.J., 574:566-574, 2002.

6. Y. Inoue. The thermal design of the polarbear-2 experiment. Master's thesis, The Graduate University for Advanced Studies, 2012.

7. Zigmund D. Kermish et al. The POLARBEAR experiment. Proceedings of SPIE mm, sub-mm, and far-IR Detectors and Instr for Astro VI 2012, pages 84521C-84521C-15, 2012.

8. John Kovac et al. Detection of polarization in the cosmic microwave background using DASI. Nature, 420:772-787, 2002.

9. T. Matsumura. A cosmic microwave background polarimeter using a superconducting magnetic bearing. $\mathrm{PhD}$ thesis, University of Minnesota, 2006.

10. Tomotake Matsumura et al. Performance of three- and five-stack achromatic half-wave plates at millimeter wavelengths. Appl. Opt., 48(19):3614-3625, Jul 2009.

11. Tomotake Matsumura et al. POLARBEAR-2 optical and polarimeter designs. Proceedings of SPIE mm, sub-mm, and far-IR Detectors and Instr for Astro VI 2012, pages 84523E-84523E-8, 2012.

12. Roger O'Brient et al. A dual-polarized broadband planar antenna and channelizing filter bank for millimeter wavelengths. Applied Physics Letters, 102(6):063506, 2013.

13. Uros Seljak et al. Signature of gravity waves in the polarization of the microwave background. Phys. Rev. Lett., 78:2054-2057, Mar 1997.

14. A. Suzuki et al. Multi-chroic dual-polarization bolometric detectors for studies of the cosmic microwave background. Journal of Low Temperature 
Physics, 2013 Submitted.

15. Aritoki Suzuki et al. Multichroic dual-polarization bolometric detectors for studies of the cosmic microwave background. Proceedings of SPIE $\mathrm{mm}$, sub-mm, and far-IR Detectors and Instr for Astro VI 2012, pages $84523 \mathrm{H}-$ 84523H-10, 2012.

16. Takayuki Tomaru et al. The POLARBEAR-2 experiment. Proceedings of SPIE mm, sub-mm, and far-IR Detectors and Instr for Astro VI 2012, pages 84521H-84521H-10, 2012. 


\section{University Library}

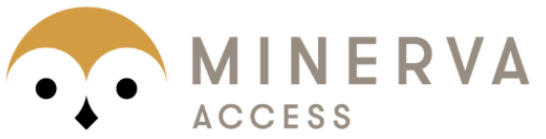

A gateway to Melbourne's research publications

Minerva Access is the Institutional Repository of The University of Melbourne

\section{Author/s:}

Suzuki, A;Ade, P;Akiba, Y;Aleman, C;Arnold, K;Atlas, M;Barron, D;Borrill, J;Chapman, S;Chinone, Y;Cukierman, A;Dobbs, M;Elleflot, T;Errard, J;Fabbian, G;Feng, G;Gilbert, A;Grainger, W;Halverson, N;Hasegawa, M;Hattori, K;Hazumi, M;Holzapfel, W;Hori, Y;Inoue, Y;Jaehnig, G;Katayama, N;Keating, B;Kermish, Z;Keskitalo, R;Kisner, T;Lee, A;Matsuda, F;Matsumura, T;Morii, H;Moyerman, S;Myers, M;Navaroli, M;Nishino, H;Okamura, T;Reichart, C;Richards, P;Ross, C;Rotermund, K;Sholl, M;Siritanasak, P;Smecher, G;Stebor, N;Stompor, R;Suzuki, J;Takada, S;Takakura, S;Tomaru, T;Wilson, B;Yamaguchi, H;Zahn, O

Title:

The POLARBEAR-2 Experiment

Date:

2014-09

\section{Citation:}

Suzuki, A., Ade, P., Akiba, Y., Aleman, C., Arnold, K., Atlas, M., Barron, D., Borrill, J., Chapman, S., Chinone, Y., Cukierman, A., Dobbs, M., Elleflot, T., Errard, J., Fabbian, G., Feng, G., Gilbert, A., Grainger, W., Halverson, N. ,... Zahn, O. (2014). The POLARBEAR-2 Experiment. Journal of Low Temperature Physics, 176, (5-6), pp.719-725. Springer Science and Business Media LLC. https://doi.org/10.1007/s10909-014-1112-x.

Persistent Link:

http://hdl.handle.net/11343/282604 